\title{
Raster Data Transfer Test Using Optigraphics Produced Data: MIL-R-28002 Type I (Raster)
}

\section{Quick Short Test Report}

\author{
June 14, 1990
}

Prepared by

Lawrence Livermore

National Laboratory

LLNL Contact

Nick Mitschkowetz

(415) 422-0582

AFLC Contact

Mel Lammers

(513) 257-3085 


\section{Contents}

1 Background and Test Objectives.................... 1

2 Test Parameters .........................................

31840 A Analysis .......................................

4 Raster Analysis ........................................

5 Conclusions and Recommendations.................. 6 


\section{Background and Test Objectives}

The DoD Computer-aided A cquisition and Logistics Support (CALS) Test Network (CTN) is conducting tests of the military standard for the Automated interchange of Technical Information, MIL-STD-1840A, and its companion suite of specifications. The CTN is a DoD sponsored confederation of voluntary participants from industry and government, managed by the Air Force Logistics Command.

The primary purpose of the CTN is to evaluate the effectiveness of the CALS standards for technical data interchange and to demonstrate the capability and operational suitability of these standards.

To this end, testing should represent the systems and applications in use by a large number of participants. Sampling a wide cross section of industry and government will gain feedback on the various interpretations of the standards and broaden the base of industry participation in the CALS initiative.

This test was conducted to allow Optigraphics to demonstrate their ability to generated a MIL-R-28002 file. The objective was to evaluate their interpretation of the MIL-R-28002 standard thereby assist the CTN in substantiating the validity of the standards or recommending changes to these standards and the references to national or international standards.

Additionally, Quick Short Test Reports (QSTRs) are intended to promote industry and government participation in the CALS initiative, developing a level of confidence in the technology and furthering mission objectives. 


\section{Test Parameters}

Test Plan: Informal data transfer between LLNL Weapons

Engineering Division (WED) and Optigraphics.

Date of

Evaluation: $\quad$ May 1, 1990

Evaluator: $\quad$ Lawrence Livermore National Laboratory

P.O. Box 808, L-542

Livermore, CA 94550

Data

Originator: Optigraphics

930 Gaydee Court

Sebastopol, CA 95472

Data

Description: $\quad$ One (1) MIL-R-28002 Type-I file was transferred over the Internet. This binary file was encoded into an ASCII text file using the UNIX "uuencode" utility. The text file was transferred, via E-mail, to mitsch@ati.tis.llnl.gov where it was decoded back to binary using the UNIX "uudecode" utility.

Data Source

System:

Optigraphics D-3100/workstation with E-size 2090-400 DPI Scanner

Evaluation

Tools Used:

SUN 3/60, UNIX

CALS Tool Box "calstb.350"

Standards

Used:

MIL-STD-1840A Notice 1 (1840A)

MIL-R-28002 Type 1 (28002) Amendment 1 


\section{A Analysis}

Data was transferred via telecommunications network directly to the CTN raster test bed, precluding any test of the transfer media or transfer format. Since MIL-STD-1840A currently only addresses 9-track magnetic tape as an exchange medium, full $1840 \mathrm{~A}$ analysis could not be conducted.

Image orientation was not considered an issue during this test. The MIL-R-28002 documentation is ambiguous in its definition of the orientation parameter. The intent of specifying raster image orientation (header record 8 "rorient:") is to allow the receiving system to render a right reading image. Images may be scanned in any orientation; it is the responsibility of the system which generates the image to indicate, through the orientation parameter, which direction the pixel path and scan line progressions are to take.

\subsection{A External Packaging Analysis}

Not applicable to this test.

\subsection{A Transmission Envelope Analysis}

Not applicable to this test.

\subsubsection{Tape Formats}

Not applicable to this test.

\subsubsection{Declaration Files}

No declaration file was delivered with the data. 


\section{Raster Analysis}

\subsection{FILE "exa.type1"}

\subsubsection{File Header Records (MIL-R-28002)}

$\begin{array}{ll}\text { 1840A header data } \\ \text { srcdocid: } & \text { exa.tif } \\ \text { dstdocid: } & \text { exa.tif } \\ \text { txtfilid: } & \text { NONE } \\ \text { sigid: } & \text { NONE } \\ \text { srcgph: } & \text { NONE } \\ \text { doccls: } & \text { UNCLASS } \\ \text { rtype: } & 1 \\ \text { rorient: } & 090,270 \\ \text { rpelcnt: } & 002304,001857 \\ \text { rdensty: } & 0200 \\ \text { notes: } & \text { Converted from an Optigraphics TIFF File. }\end{array}$

\subsubsection{File Structure (MIL-R-28002)}

header size 2048: $\quad$ OK

record size 128: $\quad$ OK

header padding $(\wedge \wedge \wedge \ldots)$ : N/A

MIL-STD-1840A references ANSI x3.27 for magnetic tape labeling and file structure. X3.27 6.3.4 specifies "Blocks within a file are padded out to the desired length by the use of 'circumflex accent' characters.....". All the files in this test used "space" characters. However, since the data was not transferred by magnetic tape, the requirement is not applicable.

First line decoding: Valid CCITT T.6 encoding octal 177777177745126075

binary 111111111111111111111111100101101110000111101

T.6 11111111111111111111111111 vertical(0) 43 blank line from beginning of image

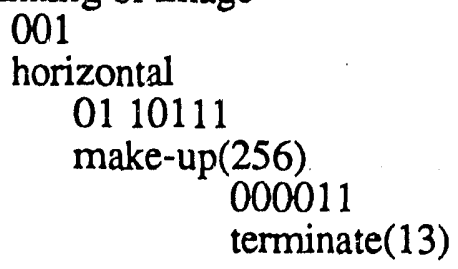

End-of-file mark decoding: Valid CCITT T.6 end of file mark octal 176000040006 binary 11111100000000000100000000000110

T.6 000000000001000000000001

$$
\text { EOF }
$$




\subsubsection{Image Presentation (CCITT Group-4)}

decompression:

The file decompressed without coding errors and an engineering drawing image was displayed.

cropped and centered: The drawing appeared complete and centered within the scanned area. The image format is nominally $8.5 \times 11$ inches with no excessive over scanning.

orthographic alignment: The aspect ratio appeared reasonable. No data objects were too "fat" or too "thin". Parallel lines appeared linear leaving no perceptible image distortion.

continuity:

The image was complete with no obvious dropouts or misalignment due to scanner read errors.

image fidelity: Image contrast was good; both wide and narrow lines were accurately captured. There was no apparent data drop out; the background was clean and free of noise. 


\section{Conclusions and Recommendations}

An image exchange with Optigraphics was accomplished via Internet Email. The Binary image file was encoded into character strings, using the SUN "uuencode" utility, to allow the source system to transfer this data over the network. Upon receipt, the destination system decoded the character strings back into binary using the SUN "uudecode" utility.

For volume data exchanges this would be an inefficient method, the test file was expanded from $19 \mathrm{~K}$ bytes to $26.3 \mathrm{~K}$ bytes during the binary to ASCII encoding. However, as an aid to accomplishing a time critical task (this file had to be transferred and processed the same day) this mechanism proved very effective. The file was transferred from Sebastopol, California, to Livermore, California, in a matter of minutes. The conventional alternative would have been to hand carry media between these two points, a 4 hour drive in one direction.

In terms of the CALS standards (MIL-STD-1840A and MIL-R-28002), this test identified an issue which can produce ambiguous data structures when other media types are implemented. MIL-STD-1840A specifies magnetic tape data structure through the ANSI X3.27 standard. That standard requires that (6.3.4 Paading of Data Blocks) "blocks within a file are padded out to the desired length by the use of circumflex accent characters".

The ambiguity manifests itself when transferring MIL-R-28002 data between differing media and different systems. Some commercial systems are sensitive to the circumflex accent character and produce a non MIL-R28002 as a result. The resulting files start the binary image data immediately after the header records rather than byte 2048 as specified.

A MIL-R-28002 file is composed of two separate sections, a 2048 byte ASCII header block followed by the group- 4 compressed image in consecutive 2048 byte binary data blocks. MIL-STD-1840A (5.1.4.4 Raster data file header records) specifies, 11 required, ASCII header records. MILSTD-1840A (5.2.1.6 Raster files) specifies the data in the first block of a raster file

shall be written with 128 byte fixed length records with a block length of 2048 bytes. The second and succeeding physical blocks of the file shall contain the image data. All the data header records shall be written in the first physical block of the file, with the block padded to the required size.

The total length of specified header data is $11 \times 128$ or 1408 total bytes, leaving 640 bytes of padding between the ASCII header data and the binary image data.

MIL-STD-1840A does not specify the character to use for padding. A vendor implementing a system which creates MIL-R-28002 files on other media such as magnetic disks is free to chose an appropriate character. When writing magnetic tape however, a vendor is required to use circumflex accent characters as required by ANSI X3.27. 
In a scenario where the CTN or a participant wants to incorporate data from disk or other magnetic media onto a MIL-STD-1840A magnetic tape for subsequent distribution, raster files would have to be dismantled and the appropriate circumflex accent padding would have to be substituted. This altering of a data file is both inefficient and dangerous to data integrity.

MIL-STD-1840A (5.2.1.6 Raster files) should state "All the data header records shall be written in the first physical block of the file, with the block padded to the appropriate size by records filled with the "space" character.

The file structure of the MIL-R-28002 test file transferred to the CTN indicates that Optigraphics can produce data files compatible with the CALS initiative. 

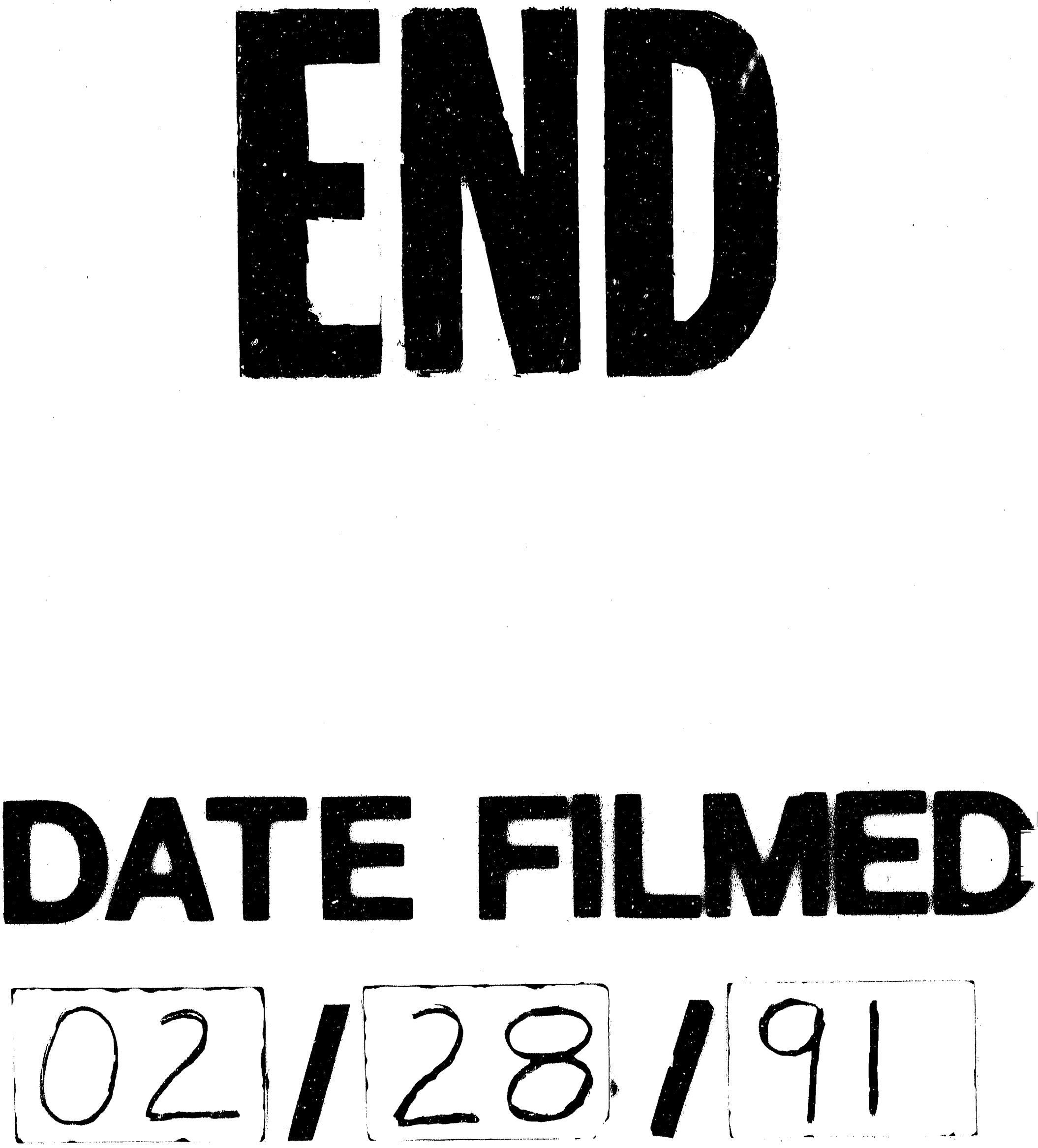
